

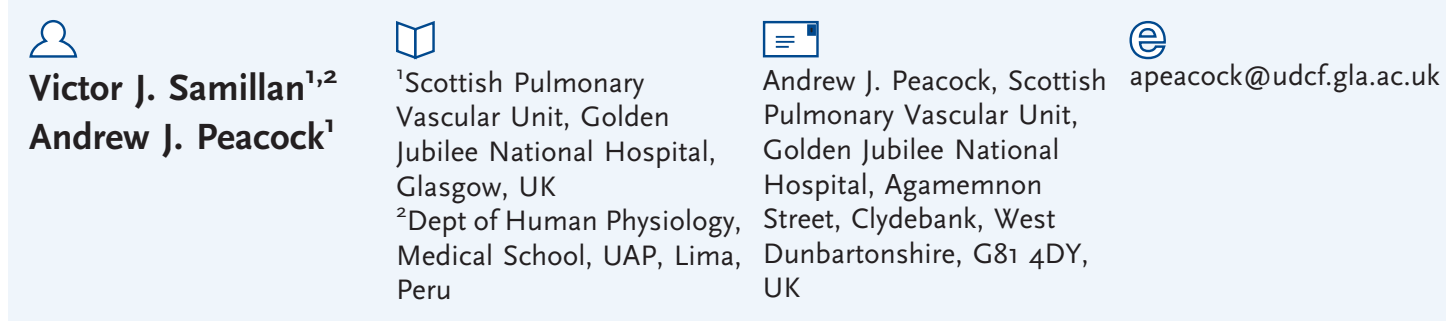

\section{State of the art: pulmonary hypertension}

\section{Key points}

- Pulmonary arterial hypertension is still a chronic and incurable disease

- The most common mutation found in familial pulmonary arterial hypertension is a defect in the bone morphogenetic protein receptor 2 gene

- Usually the final stage of the disease is right heart failure

\section{Educational aims}

- To provide definitions and diagnostic methods for pulmonary hypertension

- To provide a general classification of pulmonary hypertension

- To provide an overview of the currently available treatments

- To provide a future vision of next-generation treatments

\section{Introduction}

Pulmonary hypertension $(\mathrm{PH})$ is characterised by an elevated pulmonary arterial pressure (PAP). $\mathrm{PH}$ can occur in a number of diseases and can lead to right ventricular hypertrophy and right heart failure. This arises because the right ventricle initially adapts to the afterload with increasing wall thickness and contractility but these compensatory factors are eventually insufficient and the patients die from right heart failure. The problem may be due to abnormalities in the pulmonary arteries (and thus termed pulmonary arterial hypertension $(\mathrm{PAH})$ ) or due to other causes (and termed $\mathrm{PH}$ ).

One group of patients develop the disease due to a mutation in the bone morphogenetic protein receptor type $2(B M P R 2)$ gene. This receptor is a member of the transforming growth factor- $\beta$ family and mutations were first described in a group with familial pulmonary arterial hypertension (FPAH) $[1,2]$. Subsequently, the mutation was also found in some patients with idiopathic PAH without a history of FPAH [3].

Exposure to hypoxia is an important cause of $\mathrm{PH}$. Acute exposure to hypoxia causes $\mathrm{PH}$,
Statement of Interest Andrew J. Peacock has received grants from Actelion and Bayer, and personal fees from Actelion, Bayer and GSK, outside of this work. Victor J. Samillan has received a longterm fellowship from the European Respiratory Society. 
which is a factor in high-altitude pulmonary oedema. Exposure to chronic hypoxia occurs in the inhabitants of locations at high altitude and also in patients with lung diseases such as chronic obstructive pulmonary disease (COPD), interstitial lung disease and sleep apnoea, and leads to chronic hypoxic PH [4].

\section{Definition and classification}

The definition of $\mathrm{PH}$ is an increase of the mean PAP at rest measured during right heart catheterisation $(>25 \mathrm{mmHg})$. To make a diagnosis of $\mathrm{PAH}$, patients must also have a pulmonary capillary wedge pressure $<15 \mathrm{mmHg}$. This is an indirect method of measurement of the left atrial pressure using a balloon inflated in the pulmonary artery. Normal or low left atrial pressure excludes left heart disease. Right heart catheterisation is still necessary for the diagnosis of $\mathrm{PH}$ and needs to be standardised to ensure the same diagnostic criteria are applied to every patient. Patients with a PAP between 21 and $24 \mathrm{mmHg}$ should be monitored carefully but we need to avoid using the term "borderline PH", because these patients do not have $\mathrm{PH}$ [5].

$\mathrm{PH}$ was originally classified into two different groups, primary $\mathrm{PH}$ and secondary $\mathrm{PH}$, depending on the causes and the risk factors for developing the disease [6]. At the Second World Symposium on Pulmonary Hypertension held in Evian in 1998, a new classification was proposed, which takes into account the different features of the diseases such as haemodynamic characteristics and pathological findings. Five different groups were designated: 1) $\mathrm{PAH}$; 2) $\mathrm{PH}$ due to dysfunction of the left heart; 3) $\mathrm{PH}$ due to chronic lung disease and/or hypoxia; 4) chronic thromboembolic pulmonary hypertension (CTEPH); and 5) $\mathrm{PH}$ due to unclear mechanisms. During the recent world symposium in Nice in 2013, this classification remained broadly unchanged, except for small changes in the subclassification in each group (table 1) [7].

\section{Diagnostic methods}

The diagnosis of $\mathrm{PH}$ requires several steps, both to identify the presence of the disease and to discover its cause. One of the most difficult problems is that $\mathrm{PH}$ is often a multifactorial disease and we need to identify the principal cause. A summary of the process of diagnosis is shown in figure 1 .

\section{Clinical presentation}

The most common clinical features are breathlessness and fluid retention without other cause. Breathlessness is usually quantified, and the classification system is shown in table 2. Unfortunately, because of the late diagnosis (>2 years), the symptoms often appear when the patients are in the final stage of the disease. This may be one of the reasons why the treatments do not have a high rate of success in all patients.

\section{ECG}

An ECG often gives us the first suggestion of the disease. Classical changes are right ventricular hypertrophy and strain. Right ventricular hypertrophy is found in $87 \%$ of the patients and right axis deviation in $79 \%$ of the patients with idiopathic PAH. An ECG is not a good enough tool for determining the subgroups of this disease; therefore, additional diagnostic tests are needed [8].

\section{Echocardiography}

Echocardiography is a widely available and noninvasive tool that gives us data about the presence of $\mathrm{PH}$ (through the presence of an increased tricuspid regurgitation, jet velocity and right ventricular dilation) and also the cause (intracardiac shunts or heart abnormalities), but is insufficiently accurate as a stand-alone measurement [10].

\section{Chest radiography}

Chest radiography may suggest the presence of PH (enlarged pulmonary artery and right ventricle) and may also suggest causes (presence of lung disease or heart dysfunction), but is also insufficient for diagnosis on its own [8].

\section{Pulmonary function tests and arterial blood gases}

Pulmonary function tests and arterial blood gas measurements are useful for identifying any abnormalities suggesting obstructive (COPD or asthma) or restrictive pulmonary 
Table 1. Updated clinical classification of pulmonary hypertension ${ }^{\#}$

\section{PAH}

1.1. Idiopathic $\mathrm{PAH}$

1.2. Heritable PAH

1.2.1. BMPR2

1.2.2. ALK1, ENG, SMAD9, CAV1, KCNK3

1.2.3. Unknown

1.3. Drug and toxin induced

1.4. Associated with:

1.4.1. Connective tissue disease

1.4.2. HIV infection

1.4.3. Portal hypertension

1.4.4. Congenital heart diseases

1.4.5. Schistosomiasis

$7^{\prime}$ Pulmonary veno-occlusive disease and/or pulmonary capillary haemangiomatosis

1" Persistent $\mathrm{PH}$ of the newborn

\section{2. $\mathrm{PH}$ due to left heart disease}

2.1. Left ventricular systolic dysfunction

2.2. Left ventricular diastolic dysfunction

2.3. Valvular disease

2.4 Congenital/acquired left heart inflow/outflow tract obstruction and congenital cardiomyopathies

\section{PH due to lung diseases and/or hypoxia}

3.1. COPD

3.2. Interstitial lung disease

3.3. Other pulmonary diseases with mixed restrictive and obstructive pattern

3.4. Sleep disordered breathing

3.5. Alveolar hypoventilation disorders

3.6. Chronic exposure to high altitude

3.7. Developmental lung diseases

\section{CTEPH}

\section{PH with unclear multifactorial mechanisms}

5.1. Haematological disorders: chronic haemolytic anaemia, myeloproliferative disorders, splenectomy

5.2. Systemic disorders: sarcoidosis, pulmonary histiocytosis, lymphangioleiomyomatosis

5.3. Metabolic disorders: glycogen storage disease, Gaucher disease, thyroid disorders

5.4. Others: tumoural obstruction, fibrosing mediastinitis, chronic renal failure, segmental $\mathrm{PH}$

\#: from the Fifth World Symposium on Pulmonary Hypertension (Nice, France, 2013). Reproduced from [7] with permission from the publisher. 


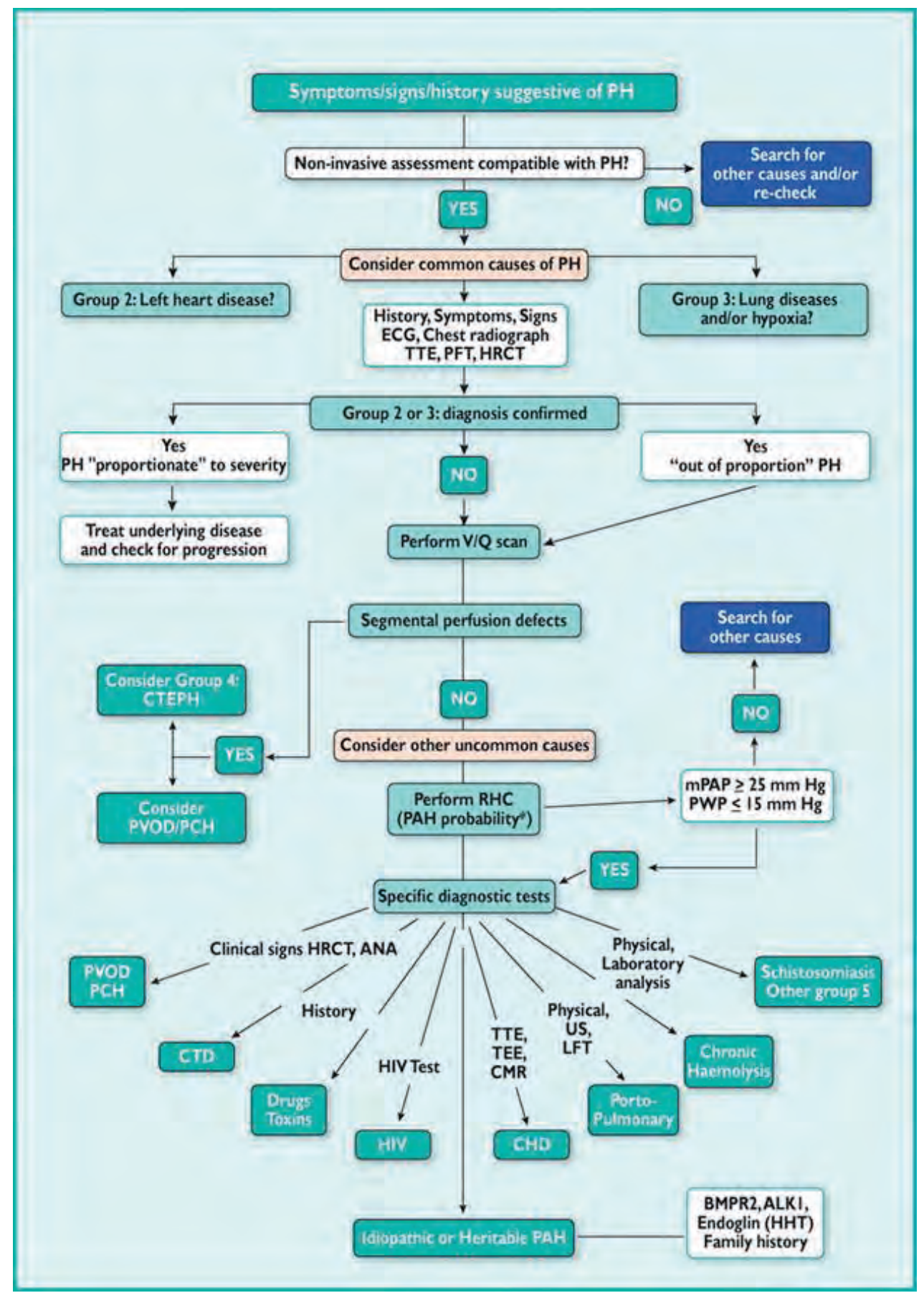

Figure 1

Diagnostic algorithm. PH: pulmonary hypertension; group: clinical group; TTE: transthoracic echocardiography; PFT: pulmonary function test; HRCT: high-resolution computed tomography; V/Q: ventilation/perfusion; CTEPH: chronic thromboembolic pulmonary hypertension; PVOD: pulmonary veno-occlusive disease; $P C H$ : pulmonary capillary haemangiomatosis; RHC: right heart catheterisation; PAH: pulmonary arterial hypertension; MPAP: mean pulmonary arterial pressure; PWP: pulmonary wedge pressure; ANA: anti-nuclear antibodies; TEE: trans-oesophageal echocardiography; CMR: cardiac magnetic resonance; US: ultrasonography; LFT: liver function test; CTD: connective tissue disease; CHD: congenital heart disease; BMPR2: bone morphogenetic protein receptor 2; ALK1: activin receptor like kinase; HHT: hereditary haemorrhagic telangiectasia. Reproduced from [8] with permission from the publisher. 
Table 2. World Health Organization functional assessment classification

Class I: Patients with PH but without resulting limitation of physical activity. Ordinary physical activity does not cause undue dyspnoea or fatigue, chest pain or near syncope.

Class II: Patients with PH resulting in slight limitation of physical activity. They are comfortable at rest. Ordinary physical activity causes undue dyspnoea or fatigue, chest pain or near syncope.

Class III: Patients with PH resulting in marked limitation of physical activity. They are comfortable at rest. Less than ordinary activity causes undue dyspnoea or fatigue, chest pain or near syncope.

Class IV: Patients with PH with inability to carry out any physical activity without symptoms. These patients manifest signs of right heart failure. Dyspnoea and/or fatigue may even be present at rest. Discomfort is increased by any physical activity.

Reproduced and modified from [9] with permission from the publisher.

disease (interstitial lung disease or chest wall abnormalities). The use of polysomnography allows a diagnosis of sleep apnoea. All these conditions can lead to $\mathrm{PH}$ (group 3) [8].

\section{Right heart catheterisation and vasoreactivity}

Right heart catheterisation is an intervention that is mandatory for confirmation of $\mathrm{PH}$. It is performed at specialised centres. The test can confirm the severity of the $\mathrm{PH}$, check for vasoreactivity and enable performance of pulmonary angiography if CTEPH is suspected. Several variables are recorded, such as cardiac output, right atrial pressure, right ventricular pressure, PAP and pulmonary artery occlusion pressure (wedge pressure) [11]. A test of vasoreactivity is performed in order to identify patients who would be candidates for calcium channel blockers. Normally, patients are given a pulmonary vasodilator such as inhaled nitric oxide and measurements are made of PAP and cardiac output. A positive test is said to have occurred when mean PAP is $<40 \mathrm{mmHg}$ with a rise or no change in cardiac output.

\section{Treatments}

Most of the treatments that we have available at the moment are focused on vasodilatation and a few are focused on reversing the vascular remodelling. All these treatments are used with the aim of improving certain outcomes, such as 6-min walking distance (6MWD) and right heart function, normalisation of the haemodynamic variables and change in biomarkers such as $\mathrm{N}$-terminal probrain natriuretic peptide $[12,13]$.
There are three main classes of treatments: 1) endothelin receptor antagonists; 2) phosphodiesterase type 5 inhibitors [13]; and 3) prostacyclins. Two new classes of drugs, the guanylate cyclase activators and prostacyclin analogues, are just becoming available or trials are finishing and the results are awaited. A summary of treatments is shown in figure 2 .

\section{Endothelin receptor antagonists}

An increase in endothelin is seen in patients with $\mathrm{PAH}$, although it is not known whether this is the cause or an effect of PAH [15]. There are two main endothelin receptors, $A$ and B. Blocking endothelin receptors blocks the vasoconstrictor and mitogenic effects of endothelin. Bosentan and macitentan are dual receptor blockers, while ambrisentan is predominantly a B receptor blocker.

\section{Ambrisentan}

Ambrisentan is a nonsulfonamide propanoic acid class drug that exerts its dominant function at the level of endothelin receptor type A [14]. In the two ARIES studies (Ambrisentan in Pulmonary Arterial Hypertension, Randomised, Double-Blind, Placebo-Controlled, Multicentre, Efficacy Study), ambrisentan resulted an improvement in 6MWD in patients with idiopathic $\mathrm{PAH}$ and $\mathrm{PAH}$ associated with HIV and connective disease [16].

\section{Bosentan}

Bosentan was the first endothelin receptor inhibitor to be synthesised. Bosentan works at the level of endothelin receptors A and B. There have been five different clinical trials of bosentan. These showed improvement in 


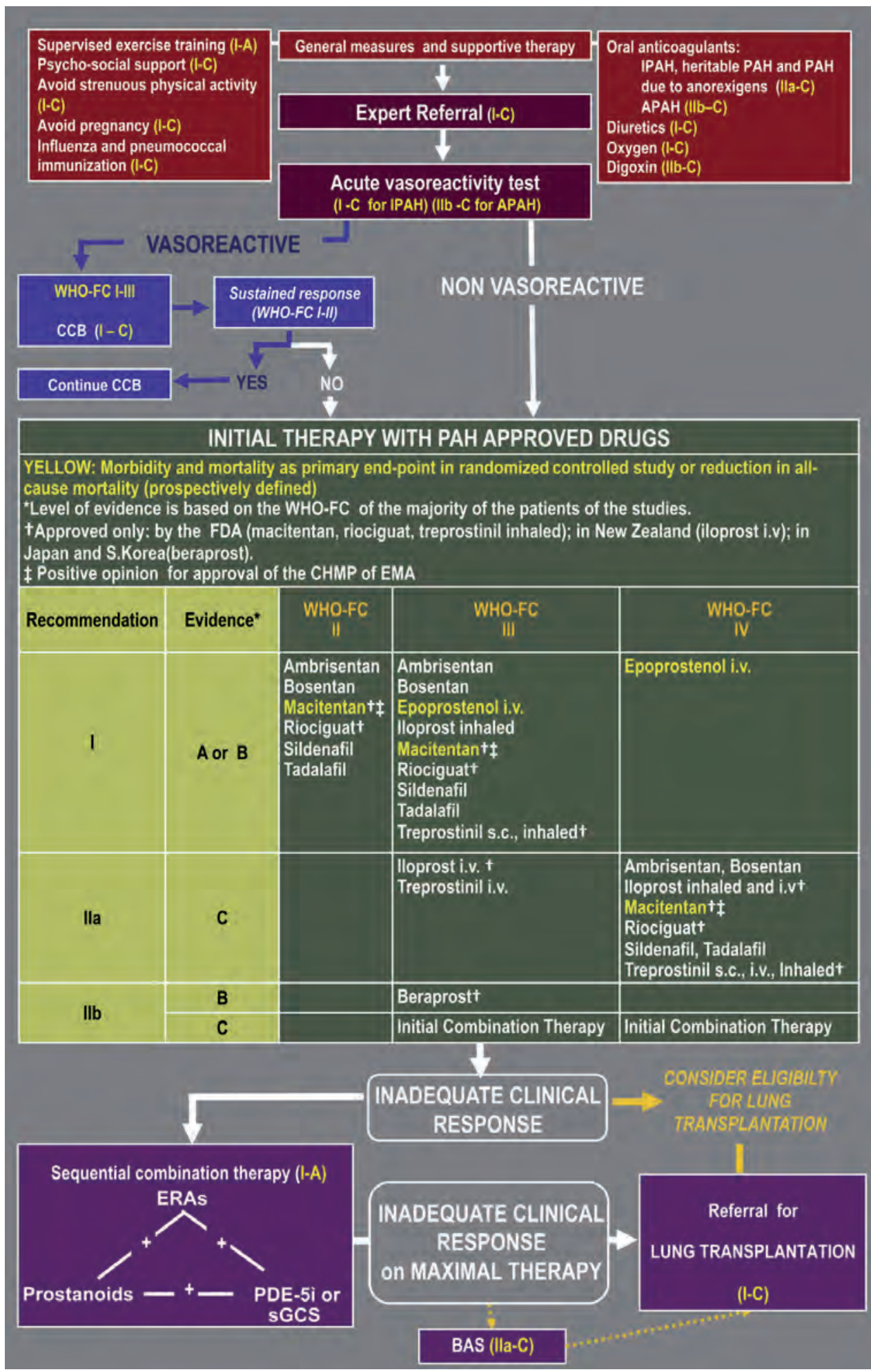

Figure 2

Treatment algorithm. IPAH: idiopathic pulmonary arterial hypertension; APAH: associated pulmonary arterial hypertension; WHO-FC: World Health Organization functional class; CCB: calcium channel blockers; FDA: US Food and Drug Administration; CHMP: Committee for Medicinal Products for Human Use; EMA: European Medicines Agency; s.c.: subcutaneous; ERA: endothelin receptor antagonist; PDE-5i: phosphodiesterase type 5 inhibitor; sGCS: soluble guanylate cyclase stimulators; BAS: balloon atrial septostomy. Reproduced from [14] with permission from the publisher. 
different clinical outcomes, such as exercise capacity, functional class, haemodynamics and time to clinical worsening [17]. In approximately $10 \%$ of patients, treatment with bosentan caused an increase of aminotransferases. For this reason, all patients must have monthly liver function tests [14].

\section{Macitentan}

Macitentan was developed using bosentan as a model. The basic molecule of bosentan was modified to improve the action and efficacy. Macitentan has a better tissue penetration and more sustained receptor binding than bosentan. Macitentan improved the morbidity/mortality end-point in patients with $\mathrm{PAH}$, in the large clinical trial SERAPHIN (Study with an Endothelin Receptor Antagonist in Pulmonary Arterial Hypertension to Improve Clinical Outcome) [18].

\section{Phosphodiesterase type 5 inhibitors}

\section{Sildenafil}

Sildenafil is the most common drug used for the treatment of $\mathrm{PH}$. This was studied in the clinical trial SUPER-1 (Sildenafil Use in Pulmonary Arterial Hypertension), leading to approval by the US Food and Drug Administration (FDA) and European Medicines Agency (EMA). Sildenafil is a potent phosphodiesterase 5 inhibitor that improves 6MWD and haemodynamics. The dose approved for $\mathrm{PAH}$ is $20 \mathrm{mg}$ three times per day. Side-effects include vasodilation (flushing, headache and epistaxis) [19, 20].

\section{Tadalafil}

Tadalafil is a once-daily phosphodiesterase 5 inhibitor that produces an improvement in the exercise capacity, symptoms, time of clinical worsening and haemodynamic variables at a dose of $40 \mathrm{mg}$ daily. The sideeffects are similar to those of sildenafil [21].

\section{Selexipag}

Selexipag is a prostacyclin analogue that attaches to the prostacyclin receptor (IP receptor). Selexipag exerts its effect through the activation of this receptor and production of cAMP. The release of this molecule produces vasorelaxation [26]. The headline results have recently been released for a large study of selexipag in PAH (GRIPHON (Prostacyclin (PGl2) Receptor agonist In Pulmonary arterial HypertensiON)).

\section{Prostacyclins}

Prostanoids are naturally released from endothelial cells and induce vasodilatation of systemic and pulmonary vasculature and also show cytoprotective and anti-proliferative effects [8].

\section{Epoprostenol}

Epoprostenol was tested in three clinical trials in patients with idiopathic PAH and patients with PAH related to scleroderma and resulted in an improvement in the different outcomes, such as exercise capacity and haemodynamics. The route of administration for epoprostenol is intravenous. For this reason, serious adverse effects often relate to the delivery system, e.g. pump malfunction, local site infection and catheter obstruction [14].

\section{Iloprost}

Iloprost is a prostacyclin analogue that can be given by i.v. or inhaled routes. The administration of inhaled iloprost was tested in the clinical trial AIR (Aerosolised Iloprost Randomised), where it was shown that inhaling iloprost six times per day $(2.5-5 \mu \mathrm{g}$ per inhalation) improved the 6MWD [22].

\section{Treprostinil}

Treprostinil is an epoprostenol analogue that is stable at room temperature. Treprostinil can be administrated subcutaneously and orally. Several clinical trials have shown the efficacy of treprostinil. Administration by the i.v. route was approved in the USA and Europe in patients with $\mathrm{PAH}$ who cannot tolerate subcutaneous administration. Currently, the oral version of treprostinil is not approved for use in patients with $\mathrm{PAH}$ [14].

\section{New classes of treatment: guanylate cyclase activators and prostacyclin analogues}

\section{Riociguat}

Riociguat is a guanylate cyclase activator that has recently been approved by the FDA in 


\section{Educational questions}

1. How high must mean PAP be to make a diagnosis of $\mathrm{PH}$ ?

a. $15 \mathrm{mmHg}$

b. $25 \mathrm{mmHg}$

c. $120 \mathrm{mmHg}$

d. $150 \mathrm{mmHg}$

2. What is the most common cause of $\mathrm{PH}$ ?

a. Cigarette smoke

b. Hypoxic lung diseases

c. Mutation of the $B M P R 2$ gene

d. Anorexigens

3. Which groups of $\mathrm{PH}$ respond to vasodilator therapy?
a. Group I
b. Group II
c. Group III
d. Group IV
e. Group V

4. Which is the most important end-point used to determine the benefits of $\mathrm{PAH}$ therapy?

a. Improvement in the right ventricle function

b. Improvement in the 6MWD

c. Improvement in the breathlessness score

d. Improvement in pulmonary haemodynamics patients with $\mathrm{PAH}$ and was launched on the market in April 2014. The difference between this drug and the phosphodiesterase 5 inhibitors is that riociguat enhances cGMP production whereas the phosphodiesterase 5 inhibitors prevent the breakdown of cGMP [23]. Riociguat works in combination with the available nitric oxide [24, 25]. Riociguat has shown anti-proliferative and anti-hypertrophy effects in different animal models.

\section{The future}

During recent years, it has become clear that, although symptoms and survival in $\mathrm{PAH}$ are much improved, we still do not have a cure for the disease. The future looks very promising, with newer pharmacological treatments that are either anti-inflammatory or anti-remodelling or both.
We now know that inflammation may contribute to $\mathrm{PH}$ and some groups of investigators are focusing on anti-inflammatory treatments. For example, some groups have been looking at cytokine pathways following the observation that interleukin-6 knockout mice do not develop $\mathrm{PH}$ [27].

We also know that remodelling of the pulmonary artery and right ventricle are the most important and challenging features when treating patients with $\mathrm{PH}$. For this reason, we need to try to find treatments to stop the remodelling. A number of laboratories are examining the cellular processes that accompany pulmonary vascular remodelling. Our own laboratory has been investigating the role of stress kinases such as mitogen-activated protein kinase kinases. In the future we may be able to block their action and reverse the remodelling [28].

\section{References}

1. Thomson JR, Machado RD, Pauciulo MW, et al. Sporadic primary pulmonary hypertension is associated with germline mutations of the gene encoding BMPR-II, a receptor member of the TGF- $\beta$ family. J Med Genet 2000; 37: 741-745.

2. Machado RD, Pauciulo MW, Thomson JR, et al. BMPR2 haploinsufficiency as the inherited molecular mechanism for primary pulmonary hypertension. Am J Hum Genet 2001; 68: 92-102.

3. Machado RD, Aldred MA, James V, et al. Mutations of the TGF- $\beta$ type II receptor BMPR2 in pulmonary arterial hypertension. Hum Mutat 2006; 27: 121-132.

4. Dempsey JA, Veasey SC, Morgan BJ, et al. Pathophysiology of sleep apnea. Physiol Rev 2010; 90: 47-112.

5. Hoeper MM, Bogaard HJ, Condliffe R, et al. Definitions and diagnosis of pulmonary hypertension. J Am Coll Cardiol 2013; 62: Suppl. 25, D42-D50.

6. Simonneau G, Galiè N, Rubin LJ, et al. Clinical classification of pulmonary hypertension. J Am Coll Cardiol 2004; 43: Suppl. 12, 5S-12S.

7. Simonneau G, Gatzoulis MA, Adatia I, et al. Updated clinical classification of pulmonary hypertension. J Am Coll Cardiol 2013; 62: Suppl. 25, D34-D41.

8. Galiè N, Hoeper MM, Humbert M, et al. Guidelines for the diagnosis and treatment of pulmonary hypertension: the Task Force for the Diagnosis and Treatment of Pulmonary Hypertension of the European Society of Cardiology (ESC) and the European Respiratory Society (ERS), endorsed by the International Society of Heart and Lung Transplantation (ISHLT). Eur Heart J 2009; 30: 2493-2537.

9. Rubin LJ, American College of Chest Physicians Diagnosis and management of pulmonary arterial hypertension: ACCP evidence-based clinical practice guidelines. Chest 2004; 126: Suppl. 1, 7S-10S

10. Fisher MR, Forfia PR, Chamera E, et al. Accuracy of Doppler echocardiography in the hemodynamic assessment of pulmonary hypertension. Am J Respir Crit Care Med 2009; 179: 615-621.

11. Paulus WJ, Tschöpe C, Sanderson JE, et al. How to diagnose diastolic heart failure: a consensus statement on the diagnosis of heart failure with normal left ventricular ejection fraction by the Heart Failure and Echocardiography Associations of the European Society of Cardiology. Eur Heart J 2007; 28: 2539-2550.

12. McLaughlin VV, Gaine SP, Howard LS, et al. Treatment goals of pulmonary hypertension. J Am Coll Cardiol 2013; 62: Suppl. 25, D73-D81.

13. Peacock AJ, Crawley S, McLure L, et al. Changes in right ventricular function measured by cardiac magnetic resonance imaging in patients receiving pulmonary arterial hypertension-targeted therapy: the EURO-MR study. Circ Cardiovasc Imaging 2014; 7: 107-114.

14. Galiè N, Corris PA, Frost A, et al. Updated treatment algorithm of pulmonary arterial hypertension. J Am Coll Cardiol 2013; 62: Suppl. 25, D60-D72.

15. Stewart DJ, Levy RD, Cernacek $P$, et al. Increased plasma endothelin-1 in pulmonary hypertension: marker or mediator of disease? Ann Intern Med 1991; 114: 464-469.

16. Galiè N, Olschewski H, Oudiz RJ, et al. Ambrisentan for the treatment of pulmonary arterial hypertension: results of the ambrisentan in pulmonary arterial hypertension, randomized, double-blind, placebocontrolled, multicenter, efficacy (ARIES) study 1 and 2. Circulation 2008; 117: 3010-3019.

17. Channick RN, Simonneau G, Sitbon O, et al. Effects of the dual endothelin-receptor antagonist bosentan in patients with pulmonary hypertension: a randomised 
placebo-controlled study. Lancet 2001; 358: 11191123.

18. Pulido T, Adzerikho I, Channick RN, et al. Macitentan and morbidity and mortality in pulmonary arterial hypertension. N Engl J Med 2013; 369: 809-818.

19. Galiè N, Ghofrani HA, Torbicki A, et al. Sildenafil citrate therapy for pulmonary arterial hypertension. N Engl J Med 2005; 353: 2148-2157.

20. Vachiery JL, Huez S, Gillies H, et al. Safety, tolerability and pharmacokinetics of an intravenous bolus of sildenafil in patients with pulmonary arterial hypertension. $\mathrm{Br} \mathrm{J}$ Clin Pharmacol 2011; 71: 289-292.

21. Pepke-Zaba J, Beardsworth A, Chan M, et al. Tadalafil therapy and health-related quality of life in pulmonary arterial hypertension. Curr Med Res Opin 2009; 25: 2479-2485.

22. Olschewski H, Simonneau G, Galiè H. Inhaled iloprost for severe pulmonary hypertension. N Engl J Med 2002; 347: 322-399.
23. Giaid A, Saleh D. Reduced expresion of endothelial nitric oxide synthase in the lungs of patients with pulmonary hypertension. N Engl J Med 1995; 333: 214-221.

24. Ghofrani HA, Simonneau G, Rubin LJ, et al. Riociguat for pulmonary hypertension. N Engl J Med 2013; 369: 2266-2268.

25. Cannon JE, Pepke-Zaba J. Riociguat for pulmonary hypertension. Expert Rev Clin Pharmacol 2014; 7: 259-270.

26. Simonneau G, Torbicki A, Hoeper MM, et al. Selexipag: an oral, selective prostacyclin receptor agonist for the treatment of pulmonary arterial hypertension. Eur Respir J 2012; 40: 874-880.

27. Savale L, Tu L, Rideau D, et al. Impact of interleukin- 6 on hypoxia-induced pulmonary hypertension and lung inflammation in mice. Respir Res 2009; 10: 6.

28. Mortimer HJ, Peacock A), Kirk A, et al. p38 MAP kinase: essential role in hypoxia-mediated human pulmonary artery fibroblast proliferation. Pulm Pharmacol Ther 2007; 20: 718-725.

\section{Suggested answers}

1. b

2. $b$

3. a

4. $a$ 